\title{
Analysis of QoS parameter in AODV a DSR in mobile Ad Hoc networks
}

\author{
Liliana Enciso Quispe and Luis Mengual Galan,
}

\begin{abstract}
Providing QoS in the context of Ad Hoc networks includes a very wide field of application from the perspective of every level of the architecture in the network. Saying It in another way, It is possible to speak about QoS when a network is capable of guaranteeing a trustworthy communication in both extremes, between any couple of the network nodes by means of an efficient Management and administration of the resources that allows a suitable differentiation of services in agreement with the characteristics and demands of every single application.

The principal objective of this article is the analysis of the quality parameters of service that protocols of routering reagents such as AODV and DSR give in the Ad Hoc mobile Networks; all of this is supported by the simulator ns-2. Here were going to analyze the behavior of some other parameters like effective channel, loss of packages and latency in the protocols of routering. Were going to show you which protocol presents better characteristics of Quality of Service (QoS) in the MANET networks.
\end{abstract}

Keywords-MANET, Ad Hoc, QoS, Parameters.

\section{INTRODUCTION}

$\mathbf{T}$ HE Ad hoc networks (MANET) $[9,14]$ are formed by a group of mobile nodes (the host, router), they can autoorganize themselves to communicate among them. All these devices that belong to Networks transmit packages and each of their nodes have to take decisions when they communicate with other nodes.The MANET Networks do not have a static infrastructure. Each of its nodes can take part in the processes of routering or authentication and distribution of resources; therefore, the management of its nodes is done in a distributed form, allowing the communication of other nodes that do not have It in a direct way, for these reasons, these types of Networks are vulnerable to assaults and risks such as: [19] denial of service (DOS), steal of service, malicious hackers, malicious code and even espionage.

When you increase nodes or points of access in the mobile networks, this becomes more insecure and therefore more complex to the insurance. Solutions already implemented by means of protocols and mechanisms of encriptation and authentication in the network exist,[18] they allow to avoid these risks, but Its still not sufficient in its entirety due to the existence of risks and threats increasingly strong [19].

In the MANET networks they can add or remove nodes without affecting the others that are connected. These

Liliana Enciso is a UTPL researcher appointed at the Search Institute of Computer Science, School of Computer Science, Technical University of Loja, Ecuador. Email: lenciso@utpl.edu.ec.

Luis Mengual is senior lecturer in the Faculty of Informatics, University of Madrid, Spain. Email: lmengual@fi.upm.es networks are capable of discovering routes and they are also prepared to recognize nodes, because they discover the presence of these automatically.

The protocols of the MANET networks, must be adaptative and to be anticiped to any change inside the network from measurement of parameters like: level of congestion, rate of mistakes, changes of used routes, etc. These parameters will give us the guidelines on the quality of service that we must offer chord to the exigency of the user or application; some of these good practices are among others: to find the most ideal route, to send only necessary messages to the channel of communication and not to consume unnecessary resources as bandwidth. With them major efficiency and an excellent performance is had in the transmission from an origination node to a recipient.

The paper is constructed this way: The section I contemplates an introduction to the mobile networks in relation to the Ad Hoc quality and safety. Section II develops the condition of the art belonging to the topic. Section III specifies relevant steps to characterize the quality in the mobile Ad Hoc networks. Section IV simulates and presents results on the differentiation of the protocols DSR and AODV for effects of the quality of the Ad Hoc networks. Finally the section V specifies conclusions on QoSs parameters analyzed in relation to the protocols AODV and DSR.

\section{A. AD HOC MOBILE NETWORKS}

II. BEEN OF THE ART

The mobile networks are indispensable for the communication between different types of mobile devices, allowing that the users should have access still when they move to and from without need to realize a new connection.

In the network Ad Hoc fig. 1, the way of communication is broadcast (sending a message from point $\mathrm{A}$ to $\mathrm{B}$, this one comes also to all the nodes, initiating in the origin and propagating to the whole network) [17]. The characteristics of a MANET are: links of limited bandwidth and changeable capacity, limitations of energy and capacity. fig.2, type of Network Ad Hoc processing in the nodes and physical limited safety. Its requirements of safety are the same that a traditional network, since they need confidentiality, integrity, authentication, not repudiation and availability $[4,8,9]$, but these characteristics make safety requirements more complex to administering. 


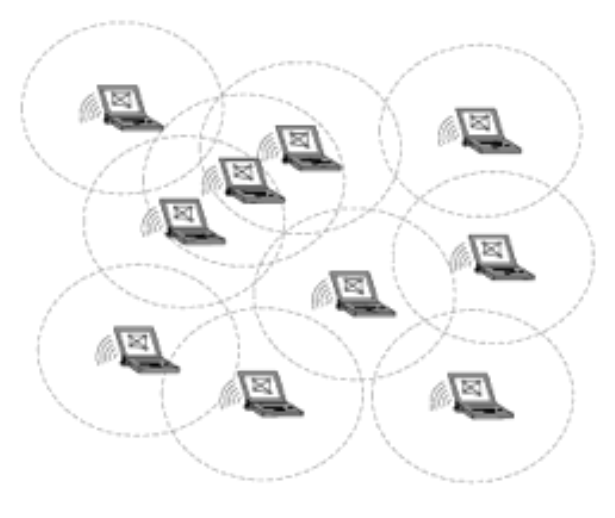

Fig. 1. Type of network Ad Hoc

Christos and Dimitrios [18] consider as examples of applications of the Ad Hoc networks: recovery of disasters, missions of search and rescue, military operations; and, between others to the distributed networks, dynamic communication of group, communication between mobile robots, etc

\section{B. CLASSIFICATION OF ROUTERING PROTOCOLS}

These protocols of agreement to their performance inside an Ad Hoc network may be classified in:

\section{1) PROACTIVE:}

Its functioning is based on tables of routes. These protocols exchange information of control among the nodes of periodic form, which allows to support current routes towards every node of the network. They are protocols that react when a new node appears or when It already is not inside the network, this exchange of information may damage the bandwidth of the application because of the overcharge of messages of control. The protocols more acquaintances are DSDV and OLSR.

\section{2) REACTIVE:}

The discovery of the route is realized only when a node wants to communicate with a concrete destination. This process concludes when the route is discovered to the target node or when all the alternatives have been looked and one has not found any route. The difficulty with these protocols is the latency on having initiated the communications, has a reaction slower to discover changes in the topology of the network. More acquaintances DSR and AODV [2].

\section{3) HYBRIDS:}

They are used for not uniform protocols. They include procedures of proactive protocols and reagents in different levels of routering; hereby, one tries to reduce the overcharge of the network generated by the proactive ones and to diminish the latency of the operations of search showed by the protocols reagents. The most known protocol is the ZRP.

\section{ROUTERING PROTOCOLS}

\section{1) PROTOCOL DSR:}

Protocol DSR (Dynamic Source Routing): .- This protocol is characterized because It has two mechanisms: Discovery of route and Maintenance of route. [12]. They are based on the concept of source routing on whom the nodes support caches that there contains the target node and the list of nodes to come to It, this list is being updated according to new routes that are learned.

In DSR when a node wants to send a package, It consults its cache to confirm if It has an available route towards this node, if he does not find it, then he will begin a discovery of route sending a RREQ (Route Request), which contains: looked delivery address, address of the node that originates the sending and an identifying only one. Every node that receives the package will check if it has a route towards the destination, but he will add his own direction in the record of routes of the package and later he will forward the package across all his links.

\section{2) PROTOCOL AODV:}

Protocol AODV (Ad Hoc On-Demand Distance Vector Routing): .- This protocol adds the concept of routering under demand; it only includes information of the nodes with which it has communication. The principal characteristic is that the nodes only exchange information of control, when they want to initiate a communication with another node.

This protocol avoids the formation of curls and of account towards the infinite, due to the fact that it supports numbers of sequence for every target node and is this characteristic the one that avoids the overcharge in the network. The table of routes is kept while they are useful and It removes itself when Its not necessary, which produces a saving in the memory and traffic in the network.

\section{QUALITY OF SERVICE OF ROUTERING IN AD HOC NETWORKS}

Parallel to the quality of service requirement is the Security that traditional networks have, It is necessary that they count with: control of access, users authentication, encriptation of information between the nodes and intruders prevention,among others. According to Christos and Dimitrios [18] these networks lack total safety in the control of physical access.The Internet is one of the principal factors that exhibits to the mobile devices to assaults like: refusal of service, injection of malicious code, interception of the network for mans assault and fraud

The problem of the Quality of Service (QoS) in mobile Ad Hoc networks has been and is one of the big problems in this type of networks. In spite of it the majority of protocols of routering for mobile Ad Hoc networks, such as AODV [1], DSR [10] are designed without considering in an explicit way 
the quality of the service of the routes that they generate. QoS of routering in Ad Hoc networks is studied and spreadin recently $[2,3,5,6,7,11,13,15,16]$.

QoS Routing needs not only to find a route of a source to a destination, but a route that QoS satisfies as final requirement, in many of the cases affected by the terms of bandwidth or delay.

Quality of the service is more difficult to guarantee in the majority of the networks and especially of the mobile Ad Hoc networks, since the bandwidth is shared between the adjacent nodes and nodes of the network or changes in the topology of the nodes when they move. This needs a wide collaboration between the nodes, to establish the route and to guarantee the necessary resources and to provide QoS. The aptitude to provide QoS, depends to a great extent on the suitable administration of the resources in the layer MAC [6].

In the mobile Ad Hoc networks, the protocols of routering like AODV (Ad Hoc On-demand Distance Vector) and DSR (Dinamic Source Routing) is very related to the quality topic and safety, since by means of these some assaults are produced, these are: Refusal of services (DOS), where the intruder by means of these protocols transmits packages RREQ (Route Request), consuming the bandwidth.

The characteristics most distinguished from an Ad Hoc or MANET networks, are their dynamic topology and the limitations of bandwidth. The functions of sending of messages of routering and managing network, are taken dynamically by the nodes, due to this the nodes assume that the communication is correct. These characteristics turn into the center of the quality problems.

The quality of service is measured basically in agreement with parameters as: throughput, latency, loss of packages, etc. With the help of the QoS it is possible to discriminate against traffic giving major or minor efficiency in the transmission of the information. When one speaks about quality of service, it is possible to say that the protocol of routering is capable of finding the way towards the destination and of delivering information in a reliable form. Aspects as loss of packages, latency in this type of networks, must be almost void especially in applications of real time[20].

\section{RELEVANT STEPS TO CHARACTERIZE THE QUALITY IN THE MOBILE AD HOC NETWORKS}

\section{A. COMPARISON BETWEEN ROUTERING PROTOCOLS}

The protocols that are object of study and simulation are AODV and DSR considered inside their classification as reactive protocol.

Important parameters of the quality of service

- Throughput.- Is the quantity of information got for the destination in a time determined across a physical or logical way in a network.
- Latency.- This parameter is measured in units of time and refers to the total passed time, since the package is transmitted by the origin until it comes to its destiny.

- Loss of packages.- This happens for the congestion of channels of transmission and because of this the packages do not reach their final destination.

The protocols AODV and DSR inside their classification are reagents, and unlike the DSR the AODV has the disadvantage that the information of routering expires. One of its characteristics is sending many small packages of control of routering (hello). On the other hand, the protocol DSR presents multiple routes, and It is one of most used in small networks, the information of routering never expires, but it is not multicast. The routering done is jump to jump, this protocol causes additional delay due to the fact that some routes are not available, but it supports routes to the destination in which traffic exists. They use a cache node, it serves to support the information of routering. It can find routes and send packages in unidirectional environments.

AODV and DSR use routering reagent known routering under demand. In this type of routering the routes to using for a certain destination only are calculated when these are necessary. These protocols try to reduce this way the overcharge generated by the messages of update of periodic routes. The principal disadvantage of the protocols reagents, is the initial delay that they introduce and that can represent a serious limitation in interactive applications that need to assure certain quality of service (ex. audio and interactive video).

\section{B. AODVs ANALYSIS (Ad Hoc On Demand Distance Vector}

\section{Characteristics}

- Only when it needs to communicate, the process of discovery of route begins.

- Major time in the delivery of packages.

- Low utilization of resources and it introduces few overcharge in the network due to the fact that it doesnt do a constant update of routes.

- It supports a locally table of routering for the already known destinies.

- It only supports the use of unidirectional links.

- It uses HELLO messages (they are used to support the information of local conectivity of a node to other one).

- They use a unique number of sequence for every destination.

- AODVs functioning depends on that every node keeps his own number of sequence updated.

\section{DSRs ANALYSIS (Dynamic Source Protocol}

\section{Characteristics}

- Has routering in the origin.

- The packages include a head-board of information about the nodes that they are going to cross. 
- It diminishes the overcharge avoiding messages of control.

- It obtains multiple possible ways towards the destination.

- Every node keeps a cache memory of routes of its neighbors.

- It detects the loss of conectividad and requests a new immediate discovery of route.

- Uses intermediate memory for the routering.

- It does discovery of routes for broadcast.

- The origin node floods the network with a plot of exploration.

- There doesnt exist any type of periodic message avoiding traffic of control in the network taking advantage of the packages for useful resources.

- It does nt use periodic HELLO packages to notify the state to its companions.

\section{Discovery of route}

On having initiated a communication between two nodes directly or across an intermediate node, begins the process of discovery of route [10], where a node can receive more of one response to the discovery of route. During the process of sending packages Route RREQ (Route Request) these record in their tables of routering the direction of the neighboring node, from which they received the broadcast package, then they establish an inverse route. If they receive additional copies of the same RREQ, these packages are rejected as soon as the destiny has been reached. The target or intermediate node, answers with a route reply unicast(RREP) to the companion from whom it received first the RREQ.

The broken links can be detected by the absence of HELLO messages. After a broken link is detected, the information is spread to all the nodes that are nearby to the node broken origin and the origin node can restart the process of discovery of route, if It needs a route to the destination.

If an origin node moves, It is capable of restarting the protocol of discovery of route to find a new route to the destination. If an intermediate node moves, this one notifies the movement to its companion and spreads a notification of link failure to its companions until the node origin is reached.

\section{SIMULATION AND RESULTS FOR EFFECTS OF CONTROL AND QUALITY OF SERVICE IN AD HOC NETWORKS}

\section{A. STAGE}

The simulation of the Ad Hoc protocols has been carried out with the tool of simulation of network ns2 (network simulator). This Ad Hoc network consists of a topology with 10 nodes, in a time of simulation of 40 seconds, where two nodes communicate using the protocol of transport TCP across intermediate nodes.

In this stage, the advantages and disavantages that the protocols AODV and DSR present are demonstrated, as it is

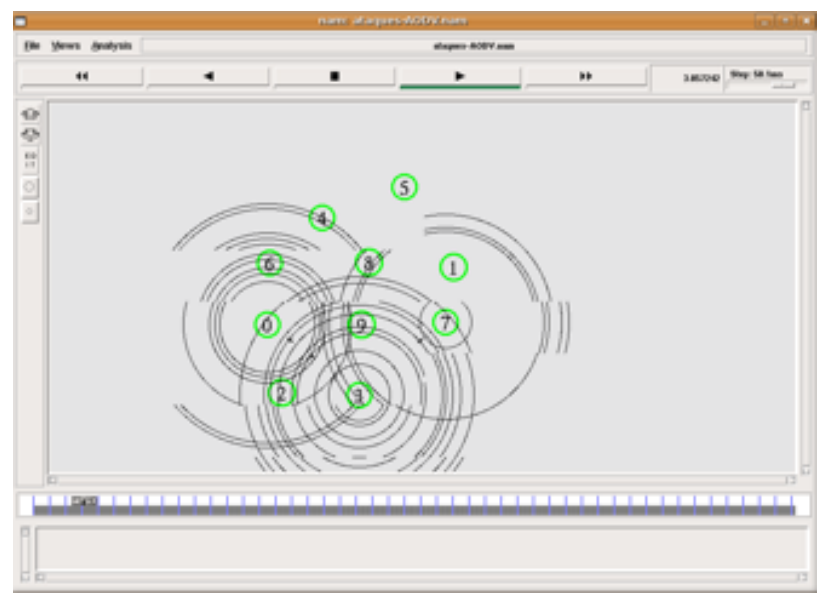

Fig. 2. Environment of simulation with NS2

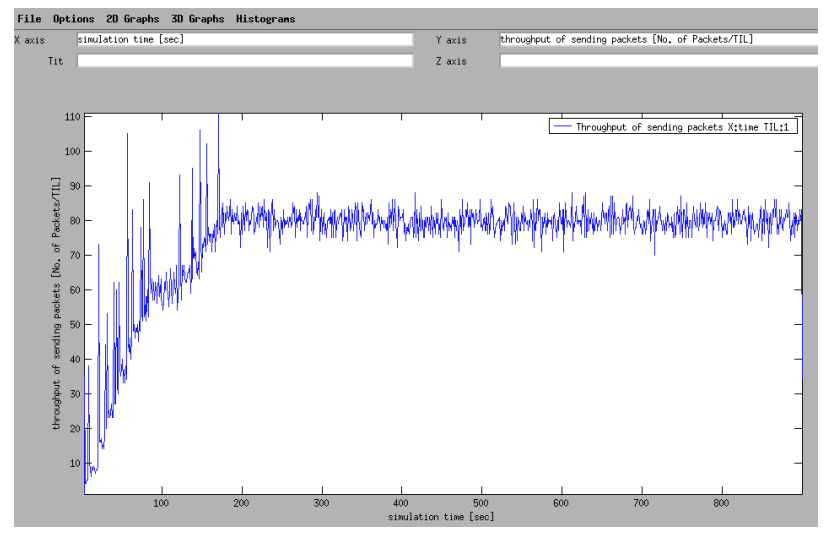

Fig. 3. Send Packets AODV

detailed in the figures $3,4,5,6,7$ and 8 .

\section{B. EVALUATION OF RESULTS}

The principal results obtained of the simulation are in the following tables I, II y III:

In the Table I, DSR presents a highest number of lost packages that AODV, but proportional to the generated

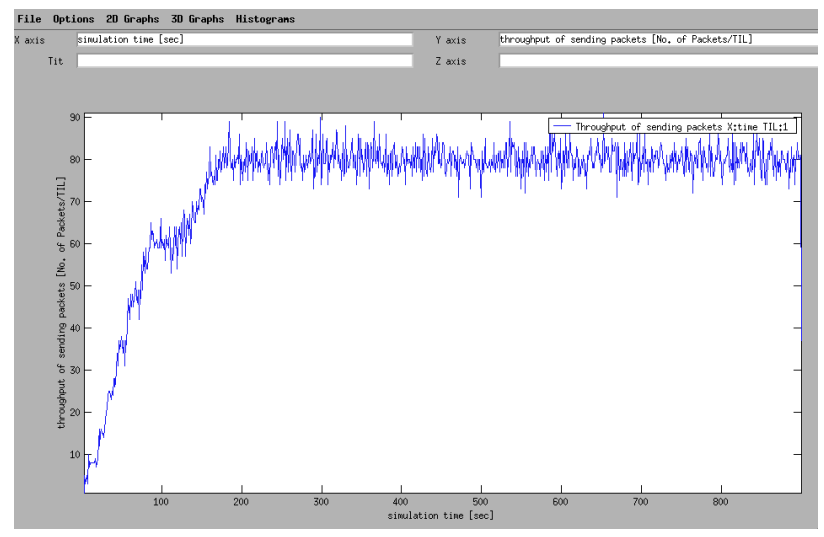

Fig. 4. Send Packets DSR 


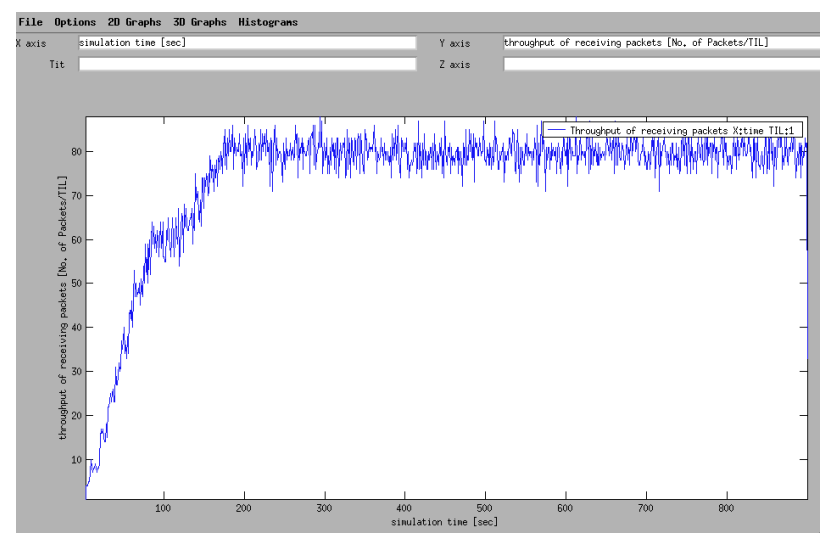

Fig. 5. Send Packets AODV

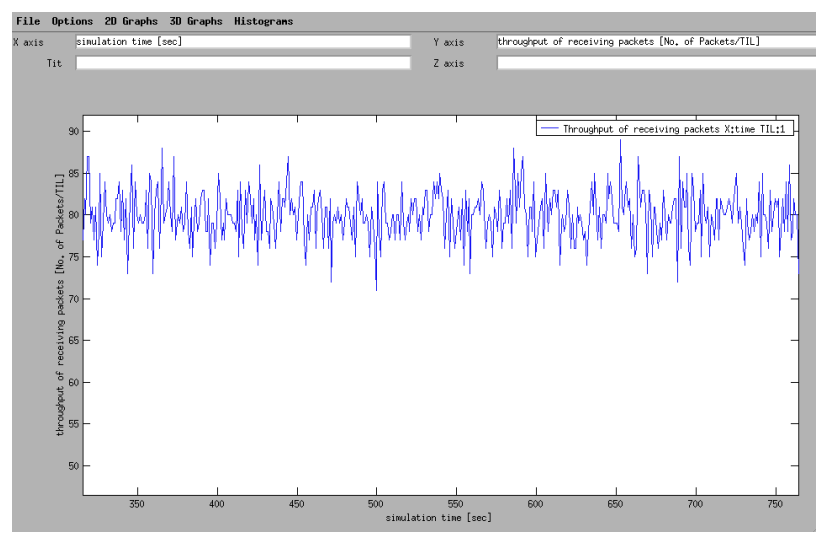

Fig. 6. Send Packets DSR

packages the lost packages is minor in DSR that in AODV , thinking that DSR generates 1390 and alone AODV 849 packages, in consequence DSR is more efficient.

The lost of packages is higher in DSR that in AODV, but DSR loses packages in agreement to the number of sendings, if we calculate mathematically DSR sends 690 packages and loses 13 packages AODV at the time on having sent 401 packages should lose 7,55 packages and like we can see the simulation throws 8 packages lost in AODV at the time we can say that they are similar. We can also notice a similar

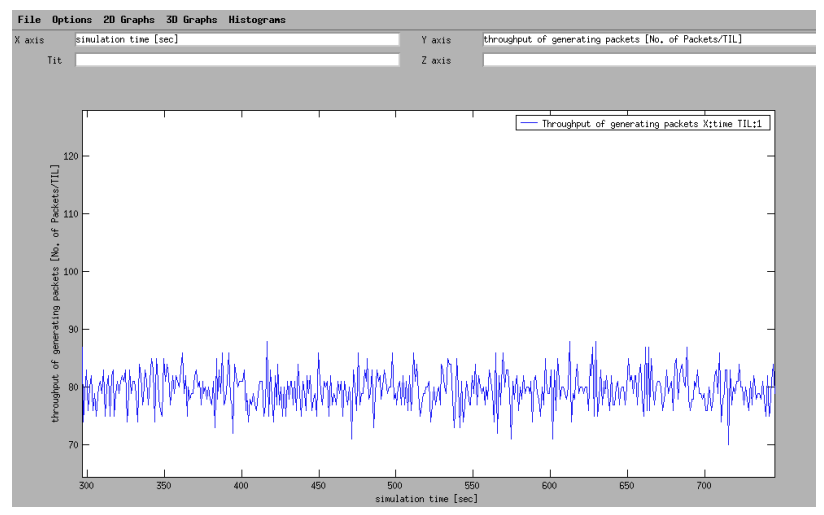

Fig. 7. Throughput AODV

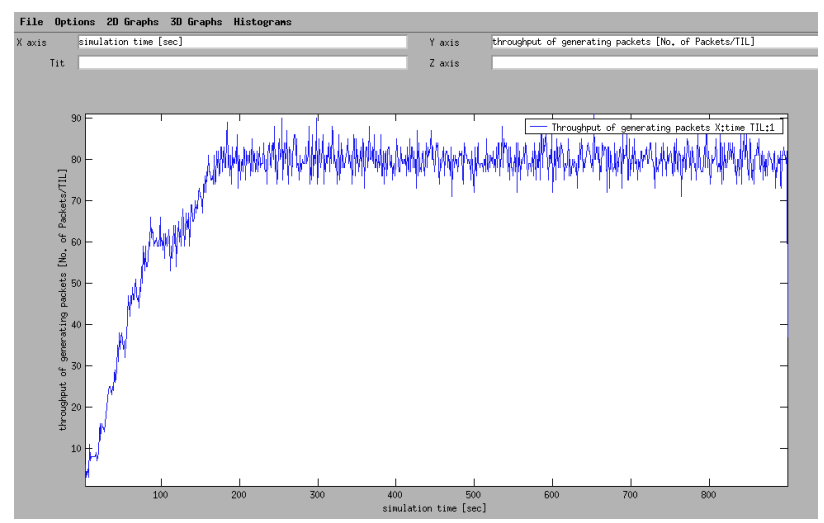

Fig. 8. Throughput DSR

\begin{tabular}{|c|c|}
\hline Options Network infornati & \\
\hline \multicolumn{2}{|c|}{ Simulation information: } \\
\hline Simulation length in & 897.4431611 \\
\hline Number of nodes: & 50 \\
\hline Number of sending nodes: & 50 \\
\hline Number of receiving nodes: & 20 \\
\hline $\begin{array}{l}\text { Number of generated packets: } \\
\text { Number of sent packets: }\end{array}$ & $\begin{array}{l}67252 \\
67176\end{array}$ \\
\hline $\begin{array}{l}\text { Number of forwarded packets: } \\
\text { Number of dropped packets: }\end{array}$ & $\begin{array}{l}1 \\
76\end{array}$ \\
\hline Number of lost packets: & 0 \\
\hline Minimal packet size: & 44 \\
\hline Maximal packet size: & 532 \\
\hline Average packet size: & 497.8465 \\
\hline Number of sent bytes: & 35332920 \\
\hline $\begin{array}{l}\text { Number of forwarded bytes: } \\
\text { Number of dropped bytes: }\end{array}$ & $\begin{array}{l}532 \\
4616 \\
\end{array}$ \\
\hline Packets dropping nodes: & $\begin{array}{lllllllll}9 & 16 & 20 & 21 & 22 & 23 & 24 & 25 & 26 \\
\end{array}$ \\
\hline \multicolumn{2}{|c|}{ Current node information: } \\
\hline Number of generated packets: & 20 \\
\hline Number of sent packets: & 20 \\
\hline Number of forwarded packets: & 0 \\
\hline Number of received packets: & 342 \\
\hline Number of dropped packets: & 0 \\
\hline Number of lost & 0 \\
\hline Number of sent & 960 \\
\hline $\begin{array}{l}\text { Number of forwarded bytes: } \\
\text { Number of received bytes: }\end{array}$ & $\begin{array}{l}0 \\
16416\end{array}$ \\
\hline $\begin{array}{l}\text { Number of dropped bytes: } \\
\text { Minimal packet size: }\end{array}$ & $\begin{array}{l}0 \\
48\end{array}$ \\
\hline Maximal packet size: & 48 \\
\hline Average packet size: & 48 \\
\hline
\end{tabular}

Fig. 9. Data generated in tracegraph for AODV

behavior in both protocols as for the erasing of packages during the transmission. See Table II.

In the simulation is observed that the delay in AODV is minor, which indicates that this protocol presents better quality of service in his routering, since the rate of lost packages is minor that DSR, and the delay that it presents in the communication between the origin and destination in his maximum and minimal point, continues being a minor in AODV.Table III.

The throughput in AODV demonstrates that the communication is established more soon than in DSR. The throughput in DSR appears 30 seconds after the simulation had started, 


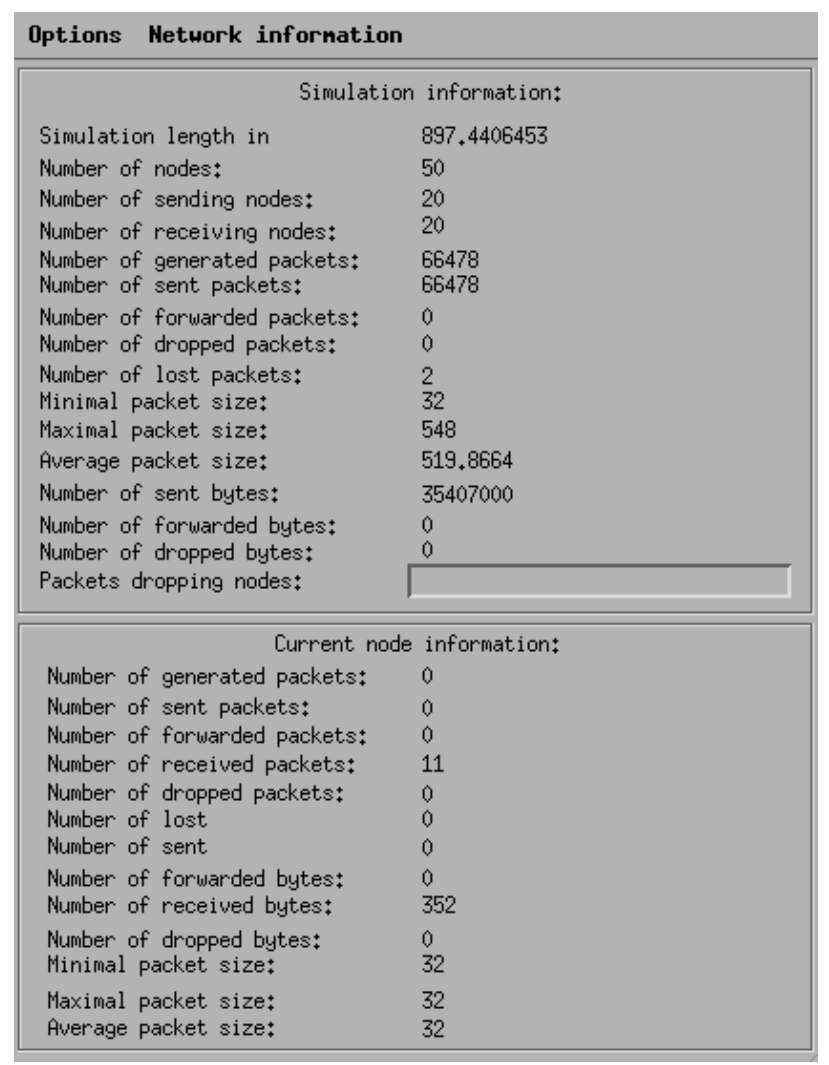

Fig. 10. Data generated in tracegraph for DSR

which indicates that in this time it manages to communicate with the destination and this value is two points during the rest of the simulation; with AODV the throughput begins at the 0 seg.Fig.7

TABLE I

Packets Management (DSR - AODV)

\begin{tabular}{l|l|l|l}
\hline Protocol & Generated packet & Sent Packets & Lost packets \\
\hline DSR & 1390 & 1390 & 23 \\
\hline AODV & 849 & 846 & 17 \\
\hline
\end{tabular}

TABLE II

COMMUNICATION FROM THE NODE 2-5)

\begin{tabular}{l|l|l|l}
\hline Protocol & Sent packet & Lost Packets & Deleted packets \\
\hline DSR & 690 & 13 & 0 \\
\hline AODV & 401 & 8 & 0 \\
\hline
\end{tabular}

TABLE III

DELAY BETWEEN THE NODE 2-5 (DSR - AODV)

\begin{tabular}{l|l|l|l}
\hline Protocol & Minimum delay & Average delay & Maximum delay \\
\hline DSR & $0.017022 \mathrm{seg}$. & $0.271257 \mathrm{seg}$. & $0.329094 \mathrm{seg}$. \\
\hline AODV & $0.004172 \mathrm{seg}$. & $0.030682 \mathrm{seg}$. & $0.193194 \mathrm{seg}$. \\
\hline
\end{tabular}

\section{CONCLUSIONS}

On the basis of the analysis, simulation and results there concludes that:
- The performance of the protocols of routering reagents depend on the stage that appears in a simulation. In normal cases as it appears in the obtained results, DSR presents better performance that AODV. This protocol in the routering with QoS produces a major performance, minor late and better effort of the protocol.

- The safety in Ad Hoc networks is not detached from the quality of service. This one represents a great challenge in the scientifical research of the computation and especially of the managers of mobile networks.

- DSR organizes enough good in networks where less than one hundred of nodes exist. In these networks DSR is specially useful and highly adaptable in small environments.

- Amongthe protocol AODV and DSR, the protocol AODV is more adapted for the quality in networks by top mobility and they adapt to big and complex environments.

- To be able to provide with QoS to the MANET networks is necessary bear in mind all the levels of the network, since it is needed of the cooperation of these to achieve the lens.

- QoSs provision in the MANET networks is a real challenge. Nevertheless, it is important to provide the MANETs de QoS to interconnect them with the wired up networks that there supports QoS (for ex. ATM, Internet with QoS, etc.) and to support applications that need processing in real time.

- AODV uses some of the advantages of the DSR protocol as mechanisms of route discovery and route maintenance, these characteristics in the process of routering serve to optimize the resources in the network and hereby to grant insurance in the quality of service. Another advantage also would be that in these protocols the decisions of routering are designated from the origin and there is no need of that the intermediate nodes support information of routering. An improvement of the DSR diminishes the size of the head-boards of the packages improves the bandwidth. A reactive protocol reduces the packages of control, reducing this way the collisions and improving again the effective bandwidth with regard to the proactive protocols. Unfortunately, it is not possible to guarantee that this protocol is the best in each and every of the networks Ad Hoc, since for certain characteristics it will be better to choose a protocol or other one. But in case of ignorance of the behavior of the nodes of the network and of the number of nodes that form her, the protocol AODV will be the most guessed right.

- For QoSs provision in Ad Hoc networks we must provide of an adaptative model of QoS where the applications have to be able to adapt to the conditions and to the availability of resources of the Ad Hoc, which are highly dynamic. These models will have to have jointly certain elements: control of admission, control of congestion, police function, some mechanism to differentiate packages and some mechanism that allows us to know the condition or state of the network. 


\section{ACKNOWLEDGMENT}

The authors gratefully acknowledge financial support from UTPL Technical University of Loja, RICS Research Institute of Computer Science and Politechnical University of Madrid.

\section{REFERENCES}

[1] Elizabeth M. Royer, Charles E. Perkins, and Samir R. Das., Ad hoc ondemand distance vector (aodv) routing 2009

[2] Elizabeth M. Royer, Charles E. Perkins, and Samir R. Das., Quality of Service for Ad hoc On-Demand Distance Vector Routing 2000

[3] Chenxi Zhu., Oos routing for mobile ad hoc networks 2006

[4] Azzedine Boukerche., Chapter 14 security and fraud detection in mobile and wireless networks. http://citeseerx.ist.psu.edu/viewdoc/summary?doi=10.1.1.99.7879.

[5] Seoung bum Lee, Gahng-Seop Ahn, Xiaowei Zhang,and Andrew T. Campbell., Insignia: An ip-based quality of service framework for mobile ad hoc networks 2000

[6] Shigang Chen and Klara Nahrstedt, Distributed quality-of-service routing in ad-hoc networks 1999.

[7] Yu ching Hsu., Qos routing in multihop packet radio environment 1998.

[8] Ignacio Soto Jose, Ignacio Moreno Ivan Vidal, Carlos Garcia., Servicios de valor aadido en redes moviles ad-hoc 2009.

[9] Kaj J.Grahn, Goran Pulkkis, Jean Sebastien Guillard., Security of mobile and wireless networks 2002.

[10] David B. Johnson and David A. Maltz., Dynamic source routing in ad hoc wireless networks pages 153181, 1996.

[11] Chunhung Richard Lin., On-demand qos routing in multihop mobile networks 2001

[12] Rajiv Misra C. R. Mandal., Performance comparison of aodv/dsr ondemand routing protocols for ad hoc networks pages 8689.2009

[13] Prasant Mohapatra, Jian Li, and Chao Gui., Qos in mobile ad hoc networks 2008

[14] Francisco Javier Ros Munoz., Evaluacion de propuestas de interconexion a internet para redes moviles ad hoc hbridas 2004.

[15] Vincent D. Park and M. Scott Corson., A highly adaptive distributed routing algorithm for mobile wireless networks 1997.

[16] T. w. Chen, J. T. Tsai, and M. Gerla Y., Qos routing performance in multihop, multimedia, wireless networks 1999

[17] Lakshmi Venkatraman y Dharma Agrawal., Strategies for enhancing routing security in protocols for mobile ad hoc networks http://citeseerx.ist.psu.edu/viewdoc/summary?doi=10.1.1.84.1303, 2003.

[18] Christos y Dimitrios., Network Security Current Satus and Future Directions 2007

[19] Tom Karygiannis y Les Owens., Wireless network security 802.11 bluetooth and handheld devices 2002

[20] Nicholas Lane, Ye Xu, Hong Lu, Shane Eisenman, Tanzeem Choudhury, Andrew Campbell., Cooperative Communities (CoCo): Exploiting Social Networks for Large-scale Modeling of Human Behavior,IEEE Pervasive Computing, Special Issue on Large-Scale Opportunistic Sensing Volume 10 Issue 4, October, 2011.

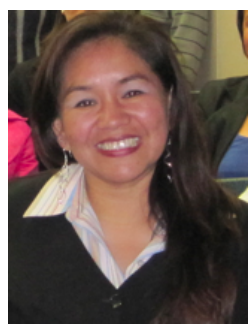

Liliana Enciso ,received a Systems Engineering degree at the Kharkov State Polytechnic University - Ukraine in 1995. Master of Science in Engineering specializing in Networking and Computer Systems and Intellectuals -1996 and PHD in progress with the Polytechnic University of Madrid - Spain. Educational Researcher is currently the Technical University of Loja - Ecuador. She is a member area networks and telecommunications unit projects and systems. He has published in the research of advanced networks and mobile networks. lenciso@utpl.edu.ec

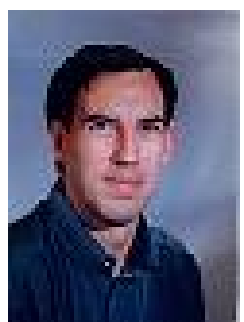

Luis Mengual Associated Professor in Languages, Computer Systems and Software Engineering Department (DLSIIS) of Computer Science School (Facultad de Informatica) of Polytechnic University of Madrid.Ph.D. in Computer Science(1998).Telecommunication Engineer Degree(1992). Teachining have experience in teaching for more than 13 years on topics related to Communications and Networks, Network Programming, Security, or Design and implementation of Distributed System. I have participated in Tempus Phare Structural Join European Project SJEP 12128-97 (TEMPUS II) helping in the field of constructing study programs and teaching methods and supervising the activities of student exchange. At the present, I am supervising Final Term Projects of students in the ERASMUS interchange program. 